\title{
Oxaliplatin Induced Neurotoxicity among Patients with Colorectal Cancer: Documentation in Medical Records-A Pilot Study
}

\author{
Jenny E. Drott' ${ }^{1}$, Hans Starkhammar ${ }^{2}$, Sussanne Börjeson' ${ }^{1}$, Carina M. Berterö ${ }^{1}$ \\ ${ }^{1}$ Department of Medical and Health Sciences, Linköping University, Linköping, Sweden \\ ${ }^{2}$ Department of Oncology, County Council of Östergötland, Linköping, Sweden \\ Email: jenny.drott@liu.se, sussanne.borjeson@liu.se, carina.bertero@.iu.se, hans.starkhammar@lio.se
}

Received 11 February 2014; revised 18 March 2014; accepted 3 April 2014

Copyright @ 2014 by authors and Scientific Research Publishing Inc.

This work is licensed under the Creative Commons Attribution International License (CC BY).

http://creativecommons.org/licenses/by/4.0/

\section{Open Access}

\begin{abstract}
Patients with colorectal cancer (CRC) can have chemotherapy with oxaliplatin postoperatively. Oxaliplatin can cause acute and chronic neurotoxicity. It is important to be aware of neurotoxic side effects so they can be documented and action taken at an early stage. The study aimed to identify and explore neurotoxic side effects documented in the medical records of patients with colorectal cancer treated with oxaliplatin-based adjuvant chemotherapy. Data in this study were medical records; presenting documentation about patients treated at the University Hospital in the south of Sweden between 2009 and 2010 . A summative content analysis approach was used to explore the neurotoxic side effects. Identification and quantification of the content of medical records were carried out by using a study-specific protocol. "Cold sensitivity" and "tingling in the hands" were the most frequently documented neurotoxicity-related terms in the medical records. This identification was followed by interpretation. Three categories were identified in the interpretive part of the study: acute, chronic, and degree of neurotoxicity. The results show the importance of awareness of neurotoxic side effects so that they can be documented and action taken at an early stage. The documentation could be more reliable if patient-reported structured measurements were used, combined with free descriptions in the medical records. Being able to follow the progression of the symptoms during and after treatment would improve patient's safety and also quality of life. The protocol that we developed and used in this review of medical records may be helpful to structure the documentation in the electronic system for documentation of neurotoxicity side effects.
\end{abstract}

\section{Keywords}

Adjuvant Oxaliplatin Chemotherapy; Colorectal Cancer; Medical Record Review; Neurotoxicity; 


\section{Summative Content Analysis}

\section{Introduction}

Colorectal cancer (CRC) is the third most common cancer type worldwide [1]. After prostate and breast cancer, CRC is the most common form of cancer in Sweden [2]. During the past five decades, a cornerstone of the treatment for CRC has been chemotherapy consisting of 5-fluorouracil (5FU) in combination with folinic acid (leucovorin). This regime has relatively low toxicity and most patients cope with treatment without severe side effects. By adding oxaliplatin the risk of relapse can be reduced by about 25 per cent compared to 5FU/leukovorin, but the neurotoxic side effects increase [2]-[6]. Common neurotoxic side effects are numbness and tingling in the hands, feet and around the mouth, muscle cramps, pain in the eye, jaw and abdomen, voice, vision, taste and sensitivity changes, and difficulty in swallowing [7]. Oxaliplatin can cause acute and chronic neurotoxicity. Studies have shown that persistent and chronic neurotoxicity problems affect patients' daily lives and health-related quality of life [8]-[12]. Acute neurotoxicity usually occurs half an hour to an hour after the start of infusion, disappears after a few days, and can develop at any dose. If the side effects are not reduced within 14 days, chronic symptomatic nerve damage can occur, leading to difficulties in swallowing, walking, writing and carrying out daily activities [9]. Permanent damage may occur without acute side effects in the initial stage [13]. Studies show that the neurotoxic side effects greatly affect the duration of chemotherapy; therefore, deciding whether the dosage of oxaliplatin should be reduced or treatment stopped for fear of debilitating and prolonged neurotoxicity is an important issue [14]-[18]. Although a number of interventions for chemotherapy-induced neurotoxicity have been tested, none of them can be recommended for clinical use due to lack of evidence [19]-[21]. Dose reduction could be useful in practice for patients who exhibit moderate or severe neurotoxicity under the treatment in order to improve the chance of therapeutic success. No formal guidelines exist for dose reduction during adjuvant therapy with oxaliplatin. The international standardized measurements such as NCI-CTC (the National Cancer Institute-Common Toxicity Criteria) can be used to grade adverse events from levels 1 - 5. Grade 2 is moderate neurotoxicity and grade 3 is severe (limiting self-care ADL) (Common Toxicity Criteria). As Cavaletti et al. show in the literature review, CTC is one of several scales [22]. Although oxaliplatin has been associated with prolonged disease-free survival time, it is interesting to focus on the patient's treatment burden. Few studies have investigated the patient's experience of neurotoxicity in qualitative studies; two studies show that patients did not expect the neurotoxicity to affect their lives to the extent that it did [8] [23]. On that point it is important that the professional's perception of patient's neurotoxicity and neurotoxicity should be documented in medical records. Consequently, there is increased concern about symptom documentation and management of neurotoxic symptoms. If the neurotoxic symptoms are under-documented in the medical records it would probably not affect the patient's quality of life but it could influence the way professionals handle the patient's problem. In order to increase the quality of care it is important to explore how the documentation of neurotoxic symptoms is carried out in the medical records. Written documentation is also a necessity when judging the quality of care provided, and it facilitates provision of continuous and individualized care by the health care professionals.

The documentation in the medical records is a secondary source and not the patient's own words/experiences; it represents the perceptions of the nurses and physicians documenting oxaliplatin-induced neurotoxicity. This study aimed to identify and explore the neurotoxic side effects documented in the medical records of patients with CRC treated with oxaliplatin-based adjuvant chemotherapy. The question was framed as follows: is it possible to identify neurotoxic side effects in the documentation/records during and after treatment?

\section{Methods}

\subsection{Design}

A summative content analysis approach including both deduction and induction was used to scrutinize medical records. The analysis started by using a study-specific protocol to allow identification and quantification of certain words related to neurotoxicity in the medical records. This quantification was used to explore usage, and at this point focus on recording the frequencies of words correlated to neurotoxicity. After this the inductive part of 
summative content analysis started including the process of interpretation of content [24] [25].

\subsection{Sample and Data Collection}

Data in this study was medical records. The records were those of men and women with a history of stage II-III CRC treated with oxaliplatin in an adjuvant setting at the University Hospital in the south of Sweden, and who had entered between 2009 and 2010. There were 180 eligible medical records on patients with CRC. Inclusion criteria were that the patients described in the medical records were at least 18 years of age, had been treated with FU/LV in combination with oxaliplatin in an adjuvant setting for stage II-III CRC, and had no neurotoxicity before oxaliplatin treatment started. Exclusion criteria were palliative treatment and known metastasis. Those records fulfilling the criteria were 61 .

A predetermined protocol to determine the documentation of patients' neurotoxic side effects was developed, influenced by the questionnaire, Oxaliplatin-Associated Neuropathy Questionnaire (OANQ) [26], see Table 1. The protocol was tested on five medical records, and since it captured the neurotoxic side effects, these medical records were included in the study.

\subsection{Ethical Considerations}

Permission to carry out the study was received by the Head of the Department and the manager responsible for electronic records, and the study and its character were judged to fulfil all the ethical criteria for research. All data were treated carefully and confidentially. Ethical approval was obtained from the Regional Ethical Review Board (record no: 2011/308-31).

\section{Table 1. Protocol to the medical record review of neurotoxic side effects.}

\begin{tabular}{|c|c|c|}
\hline Journal code & & Date \\
\hline \multicolumn{3}{|l|}{ Background factors: } \\
\hline \multicolumn{3}{|l|}{ Age } \\
\hline \multicolumn{3}{|l|}{ Sex } \\
\hline \multicolumn{3}{|l|}{ Diagnosis } \\
\hline \multicolumn{3}{|l|}{ Number of treatments } \\
\hline \multicolumn{3}{|c|}{ Interrupting treatment, cause } \\
\hline \multicolumn{3}{|c|}{ Neurotoxic side effects: } \\
\hline & Rate & Comments \\
\hline \multicolumn{3}{|l|}{$\underline{\text { Sensory }}$} \\
\hline \multicolumn{3}{|l|}{ Tingling in hands } \\
\hline \multicolumn{3}{|l|}{ Tingling in feet } \\
\hline \multicolumn{3}{|l|}{ Numbness in hands } \\
\hline \multicolumn{3}{|l|}{ Numbness in feet } \\
\hline \multicolumn{3}{|l|}{ Taste changes } \\
\hline \multicolumn{3}{|l|}{ Cold sensitivity } \\
\hline \multicolumn{3}{|l|}{ Sensitivity to heat } \\
\hline \multicolumn{3}{|l|}{ Nerve pain } \\
\hline \multicolumn{3}{|l|}{$\underline{\text { Motor }}$} \\
\hline \multicolumn{3}{|l|}{ Balance difficulty } \\
\hline \multicolumn{3}{|l|}{ Muscle weakness } \\
\hline \multicolumn{3}{|l|}{ Muscle cramps } \\
\hline \multicolumn{3}{|l|}{ Dysphagia } \\
\hline \multicolumn{3}{|l|}{ Breathing difficulties } \\
\hline Other & & \\
\hline
\end{tabular}




\subsection{Data Analysis}

Capturing of data from patients' medical records was conducted in a room in the oncology department from November to December 2011. The patients' medical records that fulfilled the inclusion criteria were marked and coded with a number from 1 to 61 . All documentation in every medical record was read twice. The medical records included documentation from all professionals in different clinical contexts. The records' text was scrutinized from the point at which the patient started adjuvant chemotherapy including oxaliplatin, and continued during and after treatment. The data corpus was 347 pages. Every specific neurotoxic side effect in the record was counted and the number of side effects was noted in the protocol. Each sentence that included neurotoxic side effects in some way was documented verbatim in the protocol. The content of the records was counted, sorted and categorized according to the protocol. The counted expressions were analysed with descriptive statistics. The verbatim statements were grouped and the categories were analysed and interpreted to understand the meaning of every category. In order to increase the validity of the findings, the analysis was subjected to peerdebriefing, and was continuously scrutinized by additional researchers (JD \& CB) [24].

\subsection{Statistical Analyses}

The primary purpose was descriptive. All descriptive statistical analyses were performed using SPSS version 19. Count frequencies, mean (SD), median (range), percent and standard deviation were used.

\section{Results}

The patients in the reviewed medical records were aged between 37 - 80 years (median 64). Of the identified patients, 35 were men (57\%) and 26 were women (43\%). Forty-five patients (74\%) had colon cancer, 15 (25\%) had rectum cancer and one patient (1\%) had both colon and rectum cancer. Patients' doses of oxaliplatin were between $120-2940 \mathrm{mg} / \mathrm{m}^{2}$ (median $1080 \mathrm{mg} / \mathrm{m}^{2}$ ). Only one patient had the maximum of 12 treatments (cycle). The mean number of treatments was $5.43(\mathrm{SD} \pm 2.36$ ). Ten patients with rectal cancer had radiation therapy before surgery.

\subsection{Identification and Quantification}

As outlined in Table 2, the three most common side effects identified in the medical records were cold sensitivity, tingling in the hands, and numbness in the hands. "Cold sensitivity" was the most repeated term in the records and seems to be a problematic side effect during chemotherapy that includes oxaliplatin. Forty-nine patients (80\%) developed neurotoxicity during chemotherapy, and for 21 patients (35\%) the neurotoxicity remained after treatment was completed. Seventeen (28\%) patients were given a reduced dose because of troublesome neurotoxicity. The dose was reduced by $30-60 \mathrm{mg} / \mathrm{m}^{2}$ from the start dose.

As outlined in Table 3, for 14 patients (23\%), neuropathy was the reason for interrupted chemotherapy. Other side effects than neurotoxicity were also documented in the medical records, e.g. nausea, diarrhoea, vomiting, jaw-locking and dehydration.

\subsection{Latent Qualitative Analysis}

The verbatim statements from the records were clustered and analysed, both in terms of description and interpretation to understand the meaning of every category. This clustering was influenced by the knowledge gained from the literature. Three categories were identified, in the text during the qualitative interpretive part of the study; acute neurotoxicity, chronic neurotoxicity and degree of neurotoxicity.

\subsubsection{Acute Neurotoxicity}

Acute neurotoxicity means symptoms that occur half an hour to an hour after the start of infusion and that usually disappear after a few days. Cold sensitivity was the most common symptom documented in 39 medical records. The term was repeated 110 times in the records under on-going treatment. No documentation was found about cold sensitivity after treatment was completed. Cold sensitivity was described as a problem with cold sensitivity in general or in specific areas, such as the mouth, throat, face, hands and feet. Cold tingling was a common description in the text. 
Table 2. Number of patients with neurotoxic side effects and frequency of words in medical record.

\begin{tabular}{ccc}
\hline & & $(\mathrm{N}=61)$ \\
\hline Neurotoxic side effects & No of patients & Frequency of words \\
\hline Cold sensitivity & $39(64 \%)$ & 110 \\
Tingling in hands & $38(62 \%)$ & 88 \\
Numbness in hands & $21(34 \%)$ & 70 \\
Numbness in feet & $20(33 \%)$ & 53 \\
Tingling in feet & $17(28 \%)$ & 40 \\
Muscle cramps & $9(15 \%)$ & 18 \\
Taste changes & $9(15 \%)$ & 10 \\
Muscle weakness & $7(11 \%)$ & 9 \\
Balance problems & $4(6 \%)$ & 7 \\
Dysphagia & $4(6 \%)$ & 4 \\
Nerve pain & $3(5 \%)$ & 4 \\
Breathing difficulties & $3(5 \%)$ & 3 \\
\hline
\end{tabular}

Table 3. Causes of interrupted treatment of oxaliplatin in the medical record (one patient completed the treatment).

\begin{tabular}{lc}
\hline Side effect & $(\mathrm{N}=61)$ \\
\hline Neurotoxicity & No of patients \\
Thrombocytopenia & $14(23 \%)$ \\
Respiratory problems, allergic reaction & $9(15 \%)$ \\
Weakness & $3(5 \%)$ \\
Severe dehydration, vomiting, diarrhea & $1(2 \%)$ \\
The patient should not have more oxaliplatin because of side effects & $3(5 \%)$ \\
No documented cause, only documented that the treatment is interrupted & $1(2 \%)$ \\
Sum of interrupted treatment; & $29(48 \%)$ \\
\hline
\end{tabular}

"Extremely cold tingling, lasted for two weeks."

"Sensitivity to cold, especially the fingers, hard to drink cold drinks."

Cold sensitivity seemed to be a major problem for the patients in the documented text and they seemed to do many things to manage and minimize the symptom. The text pointed out that patients with problems with cold sensitivity often stayed indoors, they used gloves when holding cold things, and they were careful about eating and drinking cold food and beverages. They preferred to have warm food and drinks to reduce problems with cold. In none of the records was heat sensitivity documented. Tingling in the hands was also a common problem documented in 38 of the patient records, most of them during chemotherapy but even a long time after treatment. In the medical records it showed that many patients had reported a feeling of tingling in the hands in the first days after chemotherapy, and the same was applicable for tingling in the feet. Tingling in the feet was also documented as a symptom of acute neurotoxicity associated with the on-going chemotherapy, but it was also mentioned in the text later on as a post-oxaliplatin problem, manifesting as chronic neurotoxicity.

\subsubsection{Chronic Neurotoxicity}

Chronic neurotoxicity is when symptoms remain and are not reduced within a fortnight, chronic neurotoxicity/ permanent damage may occur without acute side effects in the initial stage. A majority of the medical records showed that patients had recently completed the chemotherapy with oxaliplatin. They had reported side effects such as numbness in the hands and feet, clumsiness, and some sensory disturbance. It was a common phenomenon in the documented text that patients did not have problems during the treatment, but problems with numbness in the hands developed after completing chemotherapy. Tingling and numbness in the hands and feet was 
also a common problem but this side effect was documented both during chemotherapy and after it was completed; sometimes the delay was up to one year or more after treatment completion. Side effects were documented a long time after treatment was completed, showing that the patients had troublesome neurotoxicity for a long time. In the analysis these side effects were interpreted as symptoms of chronic neurotoxicity. In the records, chronic neurotoxicity was often documented as being associated with patient's wellbeing. This way of pointing out patients' wellbeing and adding the neurotoxic problem was interpreted and understood as minimization of chronic neurotoxicity. Some illustrations are given in these excerpts:

"Feel fine except for tingling in the feet."

"Feels really good, but still a little numbness far out in the toes."

"The patient feels completely well except for numbness in his feet."

"Feel well. Only numbness in the feet could be better."

The documentation pointed out that some side effects changed over time and became mobility problems for the patients. Muscle weakness, for example, led to balance difficulties, and dysphagia, and taste changes could result in problems with nutrition.

"Balance problems can be explained by a reduced sense of vibration of the feet and patient's description, as walking on pillows. Neurotoxicity may be suspected."

"After the last treatment, she became more or less paralyzed in the legs and could not get off the bus on her own."

"Reduced appetite due to changes in taste."

\subsubsection{Degree of Neurotoxicity}

Neurotoxicity is often reported in four different degrees when using specific scales, otherwise there are free descriptions. The degree of neurotoxicity, i.e. acute or chronic neurotoxicity, is documented in the records as a subjective judgement by the care giver. It is difficult to elaborate the degree of neurotoxicity as expressions such as some, little, a little more, much better were very often used. Some examples are given here;

"Have had a little numbness in the feet, but can play golf without problems."

"Some side effects with numbness in the finger tips."

"The tingling was much better when the dose was changed."

"A little more tingling in your hands, but better now."

No measurement, such as with scales or instruments, was used in the records to assess the degree of neurotoxicity; free descriptions were used instead. But still, a judgement was given in the record.

"Did not think she had so much trouble during the treatment however, now problems with numbness in her feet. Had mild neurotoxicity."

"Handled the adjuvant chemotherapy well, but has a lot of numbness left in the hands."

\section{Discussion}

The findings of this study of medical records show that neurotoxicity after oxaliplatin treatments in adjuvant settings after CRC is commonly documented as a problem for the patient. To our knowledge, this study is the first to explore the medical records and to include nurses' and physicians' documentation to investigate oxaliplatininduced neurotoxicity. A third of the analysed medical records described troublesome neurotoxicity that occurred after the patients had finished the cycles and completed the treatment, and health professionals seemed to minimize the problems because they recorded that the patients felt good. The reason for this neurotoxicity is a matter for speculation. For example, one reason may have been that the health professionals did not know how they could help the patients with their neurotoxicity and therefore they recorded that the patient felt well. Another reason for recording patients as being well, despite neurotoxicity, may be the lack of strategies to support the patients. Previous studies, measuring neurotoxity, have shown that some patients have persistent and chronic neurotoxicity after oxaliplatin treatment, which is in agreement with the documentation in the medical records we analysed. However, findings from other studies differ [8] [9] [11] [12]. Haller et al. [27] for example, reported grade 3 or 4 neurotoxicity with CTC (Common Toxicity Criteria) in 11\% of patients who received oxaliplatin, and chronic neurotoxicity in $5 \%$ of patients. It should be noted that the overall survival was the main end point in that study. In comparison, our findings show that the medical records of the patients in our study indicated that they had more troublesome neurotoxicity after completing the treatment. Our findings are similar 
to another study, which reported that $92 \%$ of patients had some degree of neurotoxicity during treatment, and $15.5 \%$ still had it four years after completing the therapy [28].

Our pilot study used a relatively small sample of patient's medical records, but the findings give valuable information. It was not clear in the medical records whether there was permanent nerve damage or not. Earlier studies have defined permanent nerve damage as side effects not reduced within 14 days and neurotoxicity leading to difficulties carrying out daily activities [9] [11]. In our study it was documented that the patients had problems with daily life activities when they had permanent neurotoxicity, but it was also documented that the patients felt well.

Permanent damage may occur without acute side effects in the initial stage [13], and this is confirmed in our study as well. Many patients reported problems to health professionals after they had finished treatment. These side effects would probably become permanent. Previous studies have shown that health professionals rate oxaliplatin-induced neurotoxicity less severely than is reflected in the patients' assessment of neurotoxicity [19] [21] [29]. Many factors may influence this behaviour and one reason may be that patients want to have the treatments to ensure their survival. Desire to live and belief in survival is a strong emotion in many cancer patients. Surviving includes aspects such as gratefulness and looking forward to creating a new future, and can also involve patients' coping strategies and perceived quality of life. It is an important task for nurses and physicians to make the patients confident and secure so they will inform the health professionals about the neurotoxic side effects at an early stage. Dose reduction could be one way to handle moderate or severe neurotoxicity under the treatment to improve the chance of therapeutic success and quality of life [3] [15]. Following the progression of the symptoms can improve the patient's quality of life [30] [31].

It is important that, especially during the second half of the treatment period, signs of neurotoxic symptoms caused by therapy are carefully noted. Studies show that the neurotoxic side effects are greatly affected by cumulative doses and are predominantly found in men [14] [16] [18]. These side effects may be permanent and should be weighed against the increased chance of survival that oxaliplatin gives [3] [15] [32]. In the medical records in this study it was documented that 17 (28\%) patients had a reduced dose because of troublesome neurotoxicity, but it is not clear in the records how the professionals judge when a dose reduction is necessary. There seemed not to be any consensus on criteria for making dose reductions. Heat sensitivity was not documented in any of the medical records in our study, but studies have shown that neurotoxicity can also lead to this problem [33]. Maybe one reason could be that Sweden has a cold climate, and therefore patients suffer more from cold sensitivity than in warmer countries.

There was a free description of the degree of neurotoxicity in our study, and no formal measurement was used in the records to assess this degree. The limitations of not using grading scales and symptom assessment in medical records had a negative impact on the transferability and the possibility of comparing the degree of neurotoxicity with other studies. There was a vague description of the degrees and this may have been due to the professionals' experience of the patients' problems and how they affect daily life. This could also be regarded as a limitation of this study. It is also important to be aware that the medical record is a secondary source and not the patient's own words/experience. How the health professionals handle the documentation and what they write in the record can be influenced by many factors, for example environment, time, context, stressful situations, competence of neurotoxicity and whether they recognize the side effects, and whether patients want to talk about the side effects or not. It can be a barrier to reporting symptoms and distress to the staff [34] [35]. These are all factors affecting the documentation in the records. So it could differ from hospital to hospital, and from country to country, but still the neurotoxic side effects are documented, and that is the result of our study. The results may be transferable to a similar context, but it is up to the reader to judge that, depending on the clarity when presented with the context and the sample. Summative content analysis depends on the evidence of validity, which affects credibility. The internal consistency (credibility) shows that the text evidence is consistent with the description and interpretation. In order to increase the trustworthiness of the findings the analysis was subjected to peer-debriefing and was continuously scrutinized by two additional researchers (JD \& CB).

Neurotoxic side effects often occur during treatment with oxaliplatin. Oxaliplatin can cause acute and delayed neurotoxicity. The degree of neurotoxicity in the present study is hard to compare to other studies because no standardized measurement was used to assess neurotoxicity in the medical records. Despite the existence of international standardized measurements such as CTC (Common Toxicity Criteria) and specific scales to measure oxaliplatin-induced neurotoxicity, these methods were not used by the professionals conducting the documentation in the medical records. Such an approach would be beneficial in a clinical trial setting to monitor the effi- 
cacy of interventions and to determine the true burden of neurotoxicity in oxaliplatin-treated patients. Further research is needed regarding colorectal cancer patients' experiences of neurotoxicity over time. Additional research should even have a scope of interventions, for example treating the oxaliplatin induced side effects and should also identify patients at risk of chronic neurotoxicity.

\section{Conclusion}

The results show the importance of awareness of neurotoxic side effects so that they can be documented and action taken at an early stage. Structured measurement in the electronic medical record can help nurses and physicians to document neurotoxic signs and symptoms, and even follow the progression of the symptoms during and after treatment to improve the patient's safety and also quality of life. Probably, the documentation could be more reliable if patient-reported structured measurements were used, combined with free descriptions in the medical records. Structured measurement of neurotoxicity can help nurses and physicians to document and focus on significant neurotoxic signs and symptoms, and even follow the progression of the symptoms during and after treatment to improve the patient's safety and quality of life. The protocol that we developed and used in this review of medical records may be helpful to structure the documentation in the electronic system for documentation of neurotoxicity side effects. We think that the neurotoxic protocol works well in the clinical setting and could facilitate organization of symptoms in medical records.

\section{Acknowledgements}

We thank the Department of Surgery and Oncology, Linköping University Hospital and the Division of Nursing Science at the Department of Medical and Health Sciences for financial support. We are also grateful for the financial support received at the start of this study from the Swedish Society of Nursing.

\section{References}

[1] Jemal, A., Bray, F., Center, M.M., Ferlay, J., Ward, E. and Forman, D. (2011) Global Cancer Statistics. CA: A Cancer Journal for Clinicians, 61, 69-90. http://dx.doi.org/10.3322/caac.20107

[2] Oncology Center (2008) National Health Careprogram Forcolorectal Cancer. http://www.ocsyd.se/VP-verksamhet/Gastrointest\%20ca/Vardprogr_Kolorektal-cancer081120.pdf

[3] Grothey, A. (2006) Recognizing and Managing Toxicities of Molecular Targeted Therapies for Colorectal Cancer. Oncology, 20, 21-28.

[4] Wolfe, S., Barton, D., Kottschade, L., Grothey, A. and Loprinzi, C. (2008) Chemotherapy-Induced Peripheral Neuropathy: Prevention and Treatment Strategies. European Journal of Cancer, 44, 1507-1515. http://dx.doi.org/10.1016/j.ejca.2008.04.018

[5] Provencher, S., Oeheler, C., Lavertu, S., Jolicoeur, M., Fortin, B. and Donath, D. (2010) Quality of Life and Tumor Control after Short Split-Course Chemoradiation for Anal Canal Carcinoma. Radiation Oncology, 41, 1-8.

[6] Benson III, A.B., Guillem, J.G. and Minsky, B.D. (2011) Have the Changes in Treatment of Rectal Cancer Made a Significant Difference to Our Patients? Oncology, 25, 1323-1329.

[7] Tofthagen, C., McAllister, R.D. and McMillan, S.C. (2011) Peripheral Neuropathy in Patients with Colorectal Cancer Receiving Oxaliplatin. Clinical Journal of Oncology Nursing, 15, 182-188. http://dx.doi.org/10.1188/11.CJON.182-188

[8] Bakitas, M. (2007) Background Noise. The Experience of Chemotherapy-Induced Peripheral Neuropathy. Nursing Research, 56, 323-331. http://dx.doi.org/10.1097/01.NNR.0000289503.22414.79

[9] Grenon, N. and Chan, J. (2009) Managing Toxicities Associated with Colorectal Cancer Chemotherapy and Target Therapy: A New Guide for Nurses. Clinical Journal of Oncology Nursing, 3, 285-296. http://dx.doi.org/10.1188/09.CJON.285-296

[10] Mols, F., Beijers, T., Lemmens, V., van den Hurk, C.J., Vreugdenhil, G. and van de Poll-Franse, L.V. (2013) Chemotherapy-Induced Neuropathy and Its Association with Quality of Life among 2- to 11-Year Colorectal Cancer Survivors: Results from the Population-Based PROFILES Registry. Journal of Clinical Oncology, 20, 2699-2707. http://dx.doi.org/10.1200/JCO.2013.49.1514

[11] Pietrangeli, A., Leandr, M., Terzoli, E., Jandolo, B. and Garufi, C. (2006) Persistence of High-Dose Oxaliplatin-Induced Neuropathy at Long-Term Follow-Up. European Neurology, 56, 13-16. http://dx.doi.org/10.1159/000094376

[12] Tofthagen, C., Donovan, K.A., Morgan, M.A., Shibata, D. and Yeh, Y. (2013) Oxaliplatin-Induced Peripheral Neuro- 
pathy's Effects on Health-Related Quality of Life of Colorectal Cancer Survivors. Supportive Care in Cancer, 21, 3307-3313. http://dx.doi.org/10.1007/s00520-013-1905-5

[13] Verstappen, C., Heimans, J., Hoekman, K. and Postma, T.J. (2003) Neurotoxic Complications of Chemotherapy in Patients with Cancer-Clinical Signs and Optimal Management. Drugs, 63, 1549-1563. http://dx.doi.org/10.2165/00003495-200363150-00003

[14] Beak, K.K., Lee, J., Park, S.H., Park, J.O., Park, Y.S., Lim, H.Y., Kang, W.K., Cho, Y.B., Yun, S.H., Kim, H.C., Lee, W.Y. and Chun, H.K. (2010) Oxaliplatin-Induced Chronic Peripheral Neurotoxicity: A Prospective Analysis in Patients with Colorectal Cancer. Cancer Treatment and Research, 42, 185-190. http://dx.doi.org/10.4143/crt.2010.42.4.185

[15] De Gramont, A., Buyse, M., Abrahantes, J.C., Burzykowski, T., Quinaux, E., Cervantes, A., Figer, A., Lledo, G., Flesch, M., Mineur, L., Carola, E., Etienne, P.L., Rivera, F., Chirivella, I., Perez-Staub, N., Louvet, C., André, T., Tabah-Fisch, I. and Tournigand, C. (2007) Reintroduction of Oxaliplatin Is Associated with Improved Survival in Advanced Colorectal Cancer. Journal of Clinical Oncology, 25, 3224-3229. http://dx.doi.org/10.1200/JCO.2006.10.4380

[16] Kemeny, N., Garay, C.A., Gurtler, J., Hochster, H., Kennedy, P., Benson, A., Brandt, D.S., Polikoff, J., Wertheim, M., Shumaker, G., Hallman, D., Burger, B. and Gupta, S. (2004) Randomized Multicenter Phase II Trial of Bolus plus Infusional Fluorouracil/Leucovorin Compared with Fluorouracil/Leucovorin plus Oxaliplatin as Third-Line Treatment of Patients with Advanced Colorectal Cancer. Journal of Clinical Oncology, 22, 4753-4761. http://dx.doi.org/10.1200/JCO.2004.03.119

[17] Maindrault-Goebel, F., Tournigand, C., Andre, T., Carola, E., Mabro, M., Artru, P., Louvet, C. and de Gramont, A. (2004) Oxaliplatin Reintroduction in Patients Previously Treated with Leucovorin, Fluorouracil and Oxaliplatin for Metastatic Colorectal Cancer. Annals of Oncology, 15, 1210-1214. http://dx.doi.org/10.1093/annonc/mdh305

[18] Velasco, R., Bruna, J., Briani, C., Argyriou, A., Cavaletti, G., Alberti, P., Frigeni, B., Cacciavillani, M., Lonardi, S., Cortinovis, D., Cazzaniga, M., Santos, C. and Kalofonos, H. (2013) Early Predictors of Oxaliplatin-Induced Cumulative Neuropathy in Colorectal Cancer Patients. Journal of Neurology, Neurosurgery, and Psychiatry, 85, 392-398.

[19] Wilkes, G.M. (2007) Maximising Treatment Benefit for Colorectal Cancer Patients through Evidence-Based Toxicity Management Strategies: Focus on Skin Toxicity and Peripheral Neuropathy. Colorectal Cancer Nursing, 1, 4-6.

[20] Grothey, A. (2010) Reintroduction of Oxaliplatin: A Viable Approach to the Long-Term Management of Metastatic Colorectal Cancer. Oncology, 79, 389-399. http://dx.doi.org/10.1159/000323491

[21] Hoff, P.M., Saad, E.D., Costa, F., Coutinho, A.K., Caponero, R., Prolla, G. and Gansl, R.C. (2012) Literature Review and Practical Aspects on the Management of Oxaliplatin-Associated Toxicity. Clinical Colorectal Cancer, 11, 93-100. http://dx.doi.org/10.1016/j.clcc.2011.10.004

[22] Cavaletti, G., Cornblath, D.R., Merkies, I.S., Postma, T.J., Rossi, E., Frigeni, B., Alberti, P., Bruna, J., Velasco, R., Argyriou, A.A., Kalofonos, H.P., Psimaras, D., Ricard, D., Pace, A., Galiè, E., Briani, C., Dalla Torre, C., Faber, C.G., Lalisang, R.I., Boogerd, W., Brandsma, D., Koeppen, S., Hense, J., Storey, D., Kerrigan, S., Schenone, A., Fabbri, S. and Valsecchi, M.G. (2013) The Chemotherapy-Induced Peripheral Neuropathy Outcome Measures Standardization Study: From Consensus to the First Validity and Reliability Findings. Annals of Oncology, 24, 454-462. http://dx.doi.org/10.1093/annonc/mds329

[23] Bennett, K., Park, S., Lin, C., Friedlander, M., Kiernan, M.C. and Goldstein, D. (2012) Impact of Oxaliplatin-Induced Neuropathy: A Patient Perspective. Supportive Care in Cancer, 20, 2959-2967. http://dx.doi.org/10.1007/s00520-012-1428-5

[24] Hsieh, H.F. and Shannon, S.E. (2005) Three Approaches to Qualitative Content Analysis. Qualitative Health Research, 15, 1277-1288. http://dx.doi.org/10.1177/1049732305276687

[25] Kondracki, N.L., Wellman, N.S. and Amundson, D.R. (2002) Content Analysis: Review of Methods and Their Applications in Nutrition Education. Journal of Nutrition Education and Behavior, 34, 224-230. http://dx.doi.org/10.1016/S1499-4046(06)60097-3

[26] Leonard, G.D., Wright, M.A., Quinn, M.G., Fioravanti, S., Harold, N., Schuler, B., Thomas, R.R. and Grem, J.L. (2005) Survey of Oxaliplatin-Associated Neurotoxicity Using an Interview-Based Questionnaire in Patients with Metastatic Colorectal Cancer. BMC Cancer, 5, 116-125. http://dx.doi.org/10.1186/1471-2407-5-116

[27] Haller, D.G., Catalano, P.J. and Macdonald, J.S. (2005) Phase III Study of Fluorouracil, Leucovorin, and Levamisole in High Risk Stage II and III Colon Cancer: Final Report of Intergroup 0089. Journal of Clinical Oncology, 22, 34083419.

[28] André, T., Boni, C., Navarro, M., Tabernero, J., Hickish, T., Topham, C., Bonetti, A., Clingan, P., Bridgewater, J., Rivera, F. and de Gramont, A. (2009) Improved Overall Survival with Oxaliplatin, Fluorouracil and Leucovorin as Adjuvant Treatment in Stage II or III Colon Cancer in the MOSAIC Trial. Journal of Clinical Oncology, 27, 3109-3116. http://dx.doi.org/10.1200/JCO.2008.20.6771 
[29] Postma, T.J., Heimans, J.J., Muller, M.J., Ossenkoppele, G.J., Vermorken, J.B. and Aaronson, N.K. (1998) Pitfalls in Grading Severity of Chemotherapy-Induced Peripheral Neuropathy. Annals of Oncology, 9, 739-744. http://dx.doi.org/10.1023/A:1008344507482

[30] Ramfelt, E., Severinsson, E. and Lützén, K. (2002) Attempting to Find Meaning in Illness to Achieve Emotional Coherence: The Experiences of Patients with Colorectal Cancer. Cancer Nursing, 25, 141-149. http://dx.doi.org/10.1097/00002820-200204000-00011

[31] Simpson, M.F. and Whyte, F. (2006) Patients’ Experiences of Completing Treatment for Colorectal Cancer in a Scottish District General Hospital. European Journal of Cancer Care, 15, 172-182. http://dx.doi.org/10.1111/j.1365-2354.2005.00634.x

[32] Köppler, H., Heymanns, J., Thomalla, J., Kleboth, K. and Weide, R. (2010) The Impact of New Treatment Options for Advanced Colorectal Cancer on Routine Care: Results of a Retrospective Analysis of 206 Consecutive Patients Treated in a Community-Based Oncology Group Practice in Germany. European Journal of Cancer Care, 19, 795-802. http://dx.doi.org/10.1111/j.1365-2354.2009.01111.x

[33] Attal, N., Bouhassira, D., Gautron, M., Vaillant, J.N., Mitry, E., Lepère, C., Rougier, P. and Guirimand, F. (2009) Thermal Hyperalgesia as a Marker of Oxaliplatin Neurotoxicity: A Prospective Quantified Sensory Assessment Study. Pain, 144, 245-252. http://dx.doi.org/10.1016/j.pain.2009.03.024

[34] Börjeson, S., Starkhammar, H. and Berterö, C. (2011) Common Symptoms Experienced among Patients with Colorectal Cancer, and Barriers to Reporting Symptoms or Distress: The Staff Perspective. Austral-Asian Journal of Cancer, 10, 11-20.

[35] Börjesson, S., Starkhammar, H. and Berterö, C. (2012) Common Symptoms Experienced among Patients with Colorectal Cancer: A Qualitative Part of Mixed Method Design. The Open Nursing Journal, 6, 100-107. http://dx.doi.org/10.2174/1874434601206010100 